\title{
Body composition and appetite: fat-free mass (but not fat mass or BMI) is positively associated with self-determined meal size and daily energy intake in humans
}

\author{
John E. Blundell ${ }^{1 *}$, Phillipa Caudwell ${ }^{1}$, Catherine Gibbons ${ }^{1}$, Mark Hopkins ${ }^{2}$, Erik Näslund ${ }^{3}$, \\ Neil A. King ${ }^{4}$ and Graham Finlayson ${ }^{1}$ \\ ${ }^{1}$ Biopsychology Group, Institute of Psychological Sciences, University of Leeds, Leeds LS2 9JT, UK \\ ${ }^{2}$ Sport, Health, Leisure and Nutrition, Leeds Trinity University College, Brownberrie Lane, Horsforth, Leeds, UK \\ ${ }^{3}$ Clinical Sciences (Division of Surgery), Danderyd Hospital, Stockholm, Sweden \\ ${ }^{4}$ Human Movement Studies E Institute of Health and Biomedical Innovation, Queensland University of Technology, \\ Brisbane, Australia
}

(Received 9 February 2011 - Revised 21 April 2011 - Accepted 9 May 2011 - First published online 7 July 2011)

\section{Abstract}

The idea of body weight regulation implies that a biological mechanism exerts control over energy expenditure and food intake. This is a central tenet of energy homeostasis. However, the source and identity of the controlling mechanism have not been identified, although it is often presumed to be some long-acting signal related to body fat, such as leptin. Using a comprehensive experimental platform, we have investigated the relationship between biological and behavioural variables in two separate studies over a 12-week intervention period in obese adults (total $n$ 92). All variables have been measured objectively and with a similar degree of scientific control and precision, including anthropometric factors, body composition, RMR and accumulative energy consumed at individual meals across the whole day. Results showed that meal size and daily energy intake (EI) were significantly correlated with fat-free mass (FFM, $P$ values $<0 \cdot 02-0 \cdot 05$ ) but not with fat mass (FM) or BMI ( $P$ values $0 \cdot 11-0 \cdot 45)$ (study 1, $n$ 58). In study $2(n$ 34), FFM (but not FM or BMI) predicted meal size and daily EI under two distinct dietary conditions (high-fat and low-fat). These data appear to indicate that, under these circumstances, some signal associated with lean mass (but not FM) exerts a determining effect over self-selected food consumption. This signal may be postulated to interact with a separate class of signals generated by FM. This finding may have implications for investigations of the molecular control of food intake and body weight and for the management of obesity.

Key words: Fat-free mass: Body composition: Energy intake: Meal size

The concepts of energy homeostasis and body weight regulation imply that some biological mechanism exerts control over food intake ${ }^{(1-3)}$. The precise source of this mechanism has not been confirmed, but it is widely presumed to be some signal related to body fat, such as leptin ${ }^{(4)}$, as envisaged in the lipostatic hypothesis of Kennedy ${ }^{(5)}$. In a discussion of body energy homeostasis, Keesey \& Powley ${ }^{(6)}$ identified two major sources of signals for the regulation of body energy stores: adipose tissue and the gastrointestinal tract. However, in a consideration of the dynamics of human body weight change, more emphasis is given to fat and lean tissue as separate components of body composition that influence regulation ${ }^{(7)}$. This issue is related to conjecture about the metabolic drive underlying appetite, and theorising about the role of energy expenditure (EE) in determining energy intake (EI). Following the work of Kleiber ${ }^{(8)}$, extensively used by Keesey \& Powley ${ }^{(6)}$, it is recognised that in order to exist in an energy balance, an obese person (with high energy requirements) would need to consume more energy than a lean person with lower requirements (assuming that volitional or spontaneous activity is the same). Part of the higher energy requirement of obese people is dependent on a higher RMR, and this carries an implicit acknowledgement of a link between EE and EI. Edholm et al. ${ }^{(9)}$ argued that EI was driven by EE as the process through which the energy balance was regulated. RMR is a significant component of daily EE and strongly determined by fat-free mass $(\mathrm{FFM})^{(10)}$. Edholm et al. did not detect any significant relationship between EE and EI within any single day and, surprisingly, the issue has not been investigated thoroughly with improved and more recently developed experimental methods. Moreover, there appears to be no

Abbreviations: EE, energy expenditure; EI, energy intake; FFM, fat-free mass; FM, fat mass.

*Corresponding author: Professor J. E. Blundell, email j.e.blundell@leeds.ac.uk 
definitive evidence (in humans) of relationships between FFM, fat mass (FM), RMR or some other aspect of bodily metabolism and the behavioural act of food (or energy) intake, which could throw light on the relationship between metabolism and appetite. This is partly because researchers in the field of biosciences usually operate in segmented areas of expertise. For example, researchers specialising in measuring behaviour (food intake) may have little expertise (or interest) in indirect calorimetry or body composition. Conversely, researchers who specialise in physiology may not have the expertise or interest in accurate measures of behaviour. In those studies of energy balance in which food intake has been measured accurately, the intake is often fixed (or clamped) and therefore is not free to vary as a dependent variable. Consequently, although the concept of behavioural regulation (of internal states) has been tacitly accepted among psychobiologists ${ }^{(11)}$ as a process through which energy utilisation could drive behaviour, the key variables have seldom been measured with a similar degree of accuracy. Finally, in situations in which everything has been measured accurately, researchers' hypotheses may not have drawn them to examine relationships among different classes of variables.

Within the concept of 'energy homeostasis', it is intuitive that the body's need for energy is a significant driver of food intake. Indeed, the argument is often made that the imputed mechanism functions with a high degree of precision to maintain body mass with an error of less than $1 \%{ }^{(3)}$. Importantly, FFM is the largest contributor to RMR, and RMR is normally the largest component of total energy expenditure. It therefore seems plausible that FFM, RMR or both should be related to the amount of energy that is taken in. Such a relationship could be demonstrated if daily EI and meal size could be measured accurately alongside RMR and body composition.

\section{Methods}

In two separate projects funded by the Biological and Biotechnological Science Research Council (BB/G005524/1 and $\mathrm{BB} 51 / \mathrm{B} / 05$ 079), we have developed and used an experimental platform to assess the relationship between variables of EE and measures of appetite control ${ }^{(12)}$. The methodology includes systematic measurements of imposed physical activity (five sessions per week) together with objectively and accurately monitored self-determined meals and daily EI measured in the research unit under standardised scientifically controlled conditions at intervals through a 12-week period. On specific probe days (at weeks 0, 4, 8 and 12 (study 1) or 0, 6 and 12 (study 2), body composition (air plethysmography, Bodpod, Concord, CA, USA) and RMR (indirect calorimetry, Vmax 29; Sensormedics, Yorba Linda, CA, USA) were also measured. Consequently, at corresponding time points before, during and following the 12-week intervention objective measures were made of anthropometric (body composition), physiological (RMR) and behavioural variables (meal size and daily EI) with a similar degree of precision. These procedures were carried out in overweight and obese individuals (full details of these methods have been published elsewhere $\left.{ }^{(13,14)}\right)$. In this methodology, EI was accurately and quantitatively measured on four eating occasions across the day. In study 1, four ad libitum meals were provided - breakfast, lunch, dinner and evening snack. In study 2, food intake was self-determined at three ad libitum meals; the lunch meal was fixed (in order to provide an accurate measure of postprandial satiety). Also, on the probe days in study 2 , the participants were exposed, on separate days, to high-fat or low-fat foods in order to measure meal size and daily EI under differing dietary conditions. The research was designed to investigate the extent to which active regulation of body weight (via EI and expenditure) occurs with a normal activity level (baseline) and under the challenge of imposed and supervised physical activity. The detailed measures allowed us to assess the relationships among measures of body composition (FFM and FM), obligatory energy expenditure (RMR), discretionary energy expenditure and self-determined meal sizes and daily food intake.

\section{Ethics}

The present study was conducted according to the guidelines laid down in the Declaration of Helsinki, and all procedures were approved by the NHS Leeds (West) Research Ethics Committee (no. 09/H1307/7). Written informed consent was obtained from all subjects following approval from the subject's own GP.

Table 1. Participant characteristics for both studies combined at weeks 0 and 12 and the mean difference across the 12 weeks

(Mean values and standard deviations, $n$ 92)

\begin{tabular}{|c|c|c|c|c|c|c|}
\hline & \multicolumn{2}{|c|}{ Week 0} & \multicolumn{2}{|c|}{ Week 12} & \multicolumn{2}{|c|}{ Change $0-12$} \\
\hline & Mean & SD & Mean & SD & Mean & SD \\
\hline BMI & $31 \cdot 6$ & $4 \cdot 3$ & $30 \cdot 8$ & $4 \cdot 2$ & $-1 \cdot 15^{\star \star}$ & $1 \cdot 2$ \\
\hline Body mass (kg) & 89.7 & $13 \cdot 4$ & $87 \cdot 4$ & 13.5 & $-2 \cdot 31^{\star \star}$ & $3 \cdot 3$ \\
\hline FM (kg) & $37 \cdot 2$ & 9.9 & $34 \cdot 3$ & $10 \cdot 0$ & $-2 \cdot 90^{\star \star}$ & $3 \cdot 4$ \\
\hline FFM (kg) & $52 \cdot 6$ & $9 \cdot 7$ & $53 \cdot 2$ & $9 \cdot 5$ & $+0.59^{\star \star}$ & $1 \cdot 7$ \\
\hline Body fat (\%) & $41 \cdot 2$ & $7 \cdot 9$ & $38 \cdot 9$ & $8 \cdot 3$ & $-2 \cdot 3^{\star \star}$ & $2 \cdot 6$ \\
\hline Waist circumference $(\mathrm{cm})$ & $101 \cdot 8$ & $12 \cdot 0$ & $97 \cdot 5$ & $11 \cdot 7$ & $-4 \cdot 3^{\star \star}$ & $3 \cdot 3$ \\
\hline Age (years) & $40 \cdot 8$ & $9 \cdot 2$ & & & & \\
\hline
\end{tabular}

FM, fat mass; FFM, fat-free mass.

${ }^{\star \star}$ Mean values were significantly different $(P<0.001)$. 
(a)

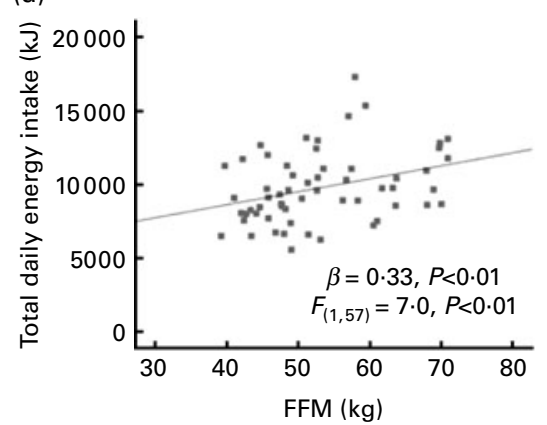

(d)

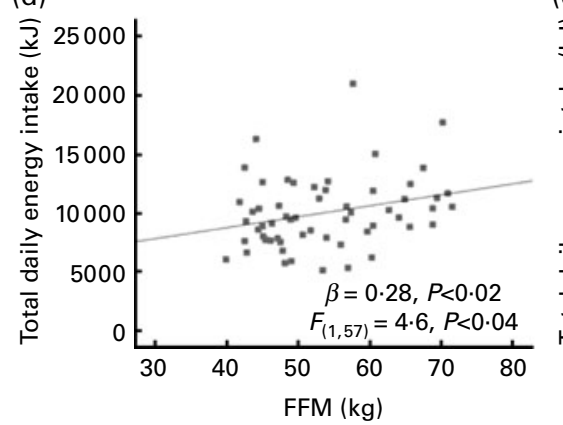

(b)

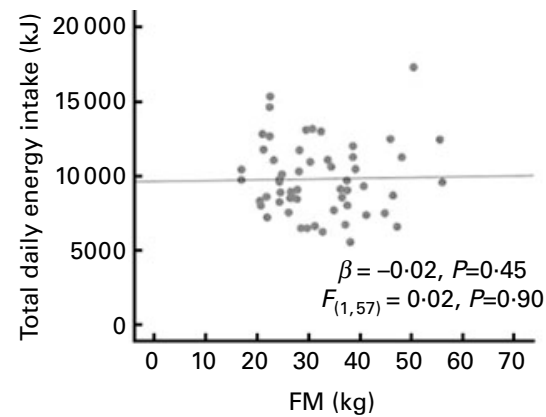

(e)

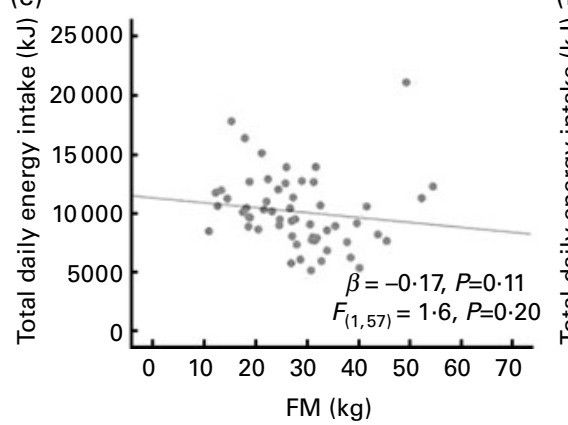

(c)

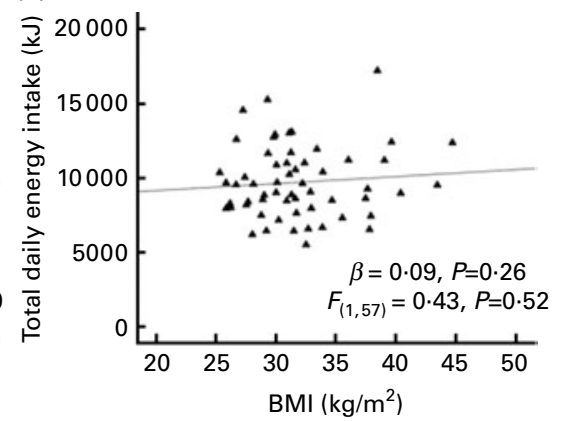

(f)

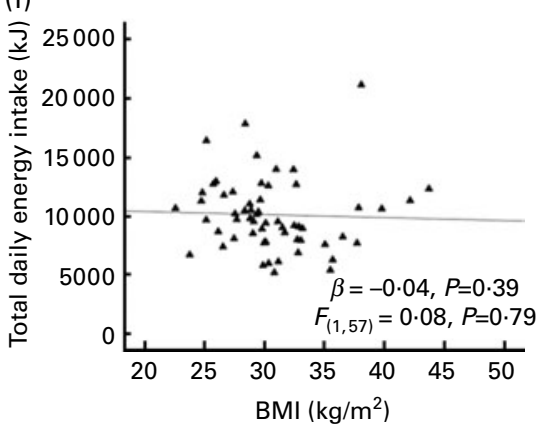

Fig. 1. Scatter plots and standardised $\beta$-coefficients to illustrate the relationship between fat mass (FM), fat-free mass (FFM) and BMI ( $n$ 58) and objectively measured total daily energy intake (total energy intake at four self-determined meals) at baseline (week 0 , before exercise) and after 12 weeks of imposed exercise. $(a-c)$ Week 0 and $(d-f)$ week 12. The relationships indicate significant correlations between total daily energy intake and FFM (but not FM or BMI). These graphs indicate the presence of an outlier participant who consumed a large amount of food energy. Because it was possible that this outlier (beyond the 95th percentile) could unduly influence the correlations, we recalculated the coefficients without the outlier. The association of FFM with daily energy intake is positive and significant and does not change the interpretation.

\section{Results and discussion}

Table 1 shows anthropometric data for the ninety-two participants who followed the 12-week programme. Data in Fig. 1 show scatter plots for the relationship between body composition variables and self-determined daily EI compiled from the four ad libitum meals consumed in study 1. Correlation coefficients indicate significant associations between FFM (but not FM or BMI) and daily EI. Table 2 shows a similar outcome for study 2 and indicates that the capacity of FFM to predict meal size occurs at baseline (week 0) and after 12 weeks of imposed physical activity. It should be noted that the regression coefficient was significant for both highfat and low-fat dietary conditions separately, and for both meal size and daily EI (data not shown). Importantly, neither BMI nor FM showed any sizeable or significant association with meal size or daily EI, suggesting that these phenotypic descriptors are not the key variables influencing the amount of energy consumed under these controlled scientific conditions. In addition to the analyses using absolute values of FFM and FM, we have also calculated the associations for FFM and FM corrected for height, as recommended recently (Dulloo et al. ${ }^{(15)}$ ). Importantly, when using FFM index and FM index, although the correlation coefficients change slightly, the significance levels do not change and the interpretation is unaltered (additional analysis supplied separately). Moreover, we have demonstrated that FFM is highly correlated with RMR ( $R$ values $=0.51-0.85, P<0 \cdot 0001)$ (confirming that the body composition measurements were valid), and RMR in turn also predicts meal size and daily EI (JE Blundell, P Caudwell, C Gibbons, M Hopkins, E Näslund, N King and $G$ Finlayson, unpublished results). Thus, any orexigenic drive arising from FFM could be mediated via EE reflected by the RMR.

One plausible interpretation of these data showing the specific association of FFM, but not fat mass (FM), with the objectively measured food intake variables is that the energy required to maintain the body's lean tissue - thus preventing wasting - determines a minimal level of EI at meals. It is worth noting that we believe that the sensitivity to detect this

Table 2. Regression coefficients for anthropometric variables and objectively measured mean dinner meal energy intake (average of highfat and low-fat dietary exposure days) $\dagger$

(Mean values with their standard errors)

\begin{tabular}{|c|c|c|c|c|c|c|}
\hline & \multicolumn{3}{|c|}{ Week $0 \ddagger$} & \multicolumn{3}{|c|}{ Week $12 \S$} \\
\hline & \multicolumn{2}{|c|}{$B$} & \multirow[b]{2}{*}{$\beta$} & \multicolumn{2}{|c|}{$B$} & \multirow[b]{2}{*}{$\beta$} \\
\hline & Mean & SE & & Mean & SE & \\
\hline Constant & 364.9 & $208 \cdot 3$ & & $102 \cdot 0$ & $217 \cdot 2$ & \\
\hline FM & $2 \cdot 8$ & 3.8 & 0.12 & 1.1 & 3.8 & 0.04 \\
\hline FFM & 9.2 & 3.4 & $0.43^{*}$ & $13 \cdot 2$ & 3.6 & $0.55^{\star \star}$ \\
\hline
\end{tabular}

${ }^{*} P<0.01,{ }^{* *} P<0.001$.

FM, fat mass; FFM, fat-free mass.

+ The relationships indicate significant correlations between average meal size and FFM (but not FM).

$\ddagger R^{2}=0.22 ; F_{(2,33)}=4.4, P<0.05$

$\S R^{2}=0.56 ; F_{(2,33)}=7.1, P<0.01$. 
relationship is due to the highly controlled laboratory conditions, which effectively prevented any possibility of overconsumption or capricious consumption arising from provocative stimuli normally present in the obesogenic environment (although food intake was free to vary). We propose that lean mass exerts an effect on EI primarily through a control of satiation (meal size). However, this claim has to be understood in the light of the fact that in our studies, meal frequency was fixed and only meal size could vary. We envisage that a learning mechanism (developing over time and prominent in the control of eating habits) contributes to the stability of this relationship. Indeed, the proposed relationship between FFM and meal size could form the basis for learned associations. This means that the possibility of reverse causality operating (meal size driving lean mass) is unlikely.

The interpretation proposed earlier has a number of implications. First, those individuals with a high FFM should have a proportionate orexigenic drive to maintain a greater minimal meal intake (i.e. they should eat more) than people with less lean tissue. This means that obese individuals (with a greater lean mass in support of adipose tissue) and people carrying a large muscle mass (field athletes, professional rugby players, swimmers etc.) should have a stronger tendency to consume large meals than smaller people. It follows that such individuals should have a greater difficulty in tolerating a dietary restriction (because the more resistant lean mass would sustain a drive for a minimal amount of food). In addition, in elderly people subject to sarcopenia, a reduced lean mass would result in diminished appetite. The role of lean mass in determining food intake can also be interpreted in the light of the landmark studies carried out on human starvation (Keys et al. ${ }^{(16)}$ ). The post-starvation recovery period has been analysed in detail (Dulloo et $a l .{ }^{(17)}$ ) and indicates a key role for FFM. Although clearly complex (and beyond the scope of the present study), an analysis of the relationship between FFM in recovery from starvation and in near normal energy balance conditions, reported in the present study, would be valuable. In addition, it is worth noting that the relationship between FFM and EI is consistent with the amino-static hypothesis put forward more than 50 years ago by Mellinkoff et $a l^{(18)}$, and with the more refined proposal of a role for a protein-stat in EI described by Millward ${ }^{(19)}$. Our data also serve to direct attention away from fat stores and onto other aspects of body composition as determinants of appetite control. This is in keeping with a widening ergostatic function of additional adipostatic hormones in energy homeostasis as envisaged by Fruhbeck \& Gomez-Ambrosi ${ }^{(20)}$. The idea of FFM creating an orexigenic or ergostatic drive has also been proposed in other depictions of the regulatory system $^{(21)}$. It can also be noted that hormones and factors collectively called 'adipokines' exert effects not only on adipose tissue but also on skeletal muscle ${ }^{(22)}$, and that differential expression of genes and pathways intrinsic to skeletal muscle may also be involved ${ }^{(23)}$, adding further possible mechanisms to support a link between energy homeostasis and FFM.

The relationships between FFM and meal size reported earlier have been disclosed in two separate projects carried out under two separate Biotechnology and Biological Sciences
Research Council programmes in consecutive 3-year periods. The relationships therefore are reproducible and seem reliable. What is surprising to us is why these relationships did not become apparent in earlier research studies. Perhaps, it has been assumed that food intake is proportional to lean mass, and the relationship did not have to be demonstrated. On the other hand, the concept may have been dismissed in favour of the widely held proposed role of adipose tissue in appetite control and weight regulation as envisaged in the lipostatic hypothesis. In our studies, body composition was measured by air plethysmography rather than dual-energy absorptiometry because of the need for multiple measurements over time in our participants, but we can find no way in which the use of a particular technique could have accounted for the results demonstrated. Clearly, the associations reported in the present study should be confirmed in other laboratories, but using objective measurements of physiological and behavioural variables under similar closely controlled scientific conditions. Importantly, food intake must be measured quantitatively and objectively under controlled conditions. Self-reports of food intake, by whatever method, are not sufficiently accurate. A strong argument from these findings suggests that for investigations carried out under these experimental conditions, some signal associated with FFM (effectively lean tissue) exerts a determining effect over the amount of food that is freely consumed. A weaker argument is that FFM and EI are merely correlated, but this is still interesting. Our hypothesis is that lean mass sets a lower limit for self-determined meal size and therefore maintains a minimal level of EI. This finding has implications for the investigation of the molecular control of food intake and body weight, and suggests that more attention should be given to lean mass, as well as to adipose tissue, in the management of obesity.

\section{Acknowledgements}

The present study was supported by Biotechnology and Biological Sciences Research Council grants: BBS/B/05079 Individual Variability and Characterisation of Compensatory Responses to exercise Interventions; BB/G005524/1 - Drivers of eating behaviour during chronic overconsumption, International Standards Randomized Control Trial Number ISRCTN47291569. The authors declare no conflict of interest. P. C., C. G. and M. H. performed the experimental studies, prepared the figure and tables, and contributed to the manuscript. G. F., N. A. K. and E. N. supervised the experimental studies and contributed to the manuscript. J. E. B. supervised the studies, prepared the first draft of the manuscript and finalised the text.

\section{References}

1. Druce M \& Bloom SR (2003) Central regulation of food intake. Curr Opin Clin Nutr Metab Care 6, 361-367.

2. Korner J \& Leibel RL (2003) To eat or not to eat - how the gut talks to the brain. $N$ Engl J Med 349, 941-948.

3. Woods SC (2005) Signals that influence food intake and body weight. Physiol Behav 86, 709-716. 
4. Friedman JM \& Halaas JL (1998) Leptin and the regulation of body weight in mammals. Nature 395, 763-770.

5. Kennedy GC (1953) The role of depot fat in the hypothalamic control of food intake in the rat. Proc $R$ Soc Lond $B$ 140, 578-592.

6. Keesey RE \& Powley TL (2008) Body energy homeostasis. Appetite 51, 442-445.

7. Chow CC \& Hall KD (2008) The dynamics of human body weight change. PLoS Comput Biol 4, e1000045.

8. Kleiber M (1947) Body size and metabolic rate. Physiol Rev 15, 511-541.

9. Edholm OG, Fletcher JG, Widdowson EM, et al. (1955) The energy expenditure and food intake of individual men. $\mathrm{Br} \mathrm{J}$ Nutr 9, 286-300.

10. Welle S \& Nair KS (1990) Relationship of metabolic rate to body composition and protein turnover. Am J Physiol Endocrinol Metab 258, E990-E998.

11. Richter C (1956) Loss of appetite for alcohol and alcoholic beverages produced in rats by treatment with thyroid preparations. Endocrinology 59, 472-478.

12. Caudwell P, Gibbons C, Hopkins M, et al. (2011) The influence of physical activity on appetite control: an experimental system to understand the relation between exercise-induced energy expenditure and energy intake. Proc Nut Soc 70, $171-180$.

13. King N, Hopkins M, Caudwell $\mathrm{P}$, et al. (2008) Individual variability following 12 weeks of supervised exercise: identification and characterization of compensation for exerciseinduced weight loss. Int J Obes (London) 32, 177-184.

14. King N, Caudwell P, Hopkins M, et al. (2009) Dual-process action of exercise on appetite control: increase in orexigenic drive but improvement in meal-induced satiety. Am J Clin Nutr 90, 921-927.

15. Dulloo AG, Jacquet J, Solinas G, et al. (2010) Body comopsition phenotypes in the pathways to obesity and metabolic syndrome. Int J Obes (Lond) 34, Suppl. 2, S4-S17.

16. Keys A, Brozek J, Henschel A, et al. (1950) The Biology of Human Starvation. Minneapolis: University of Minnesota Press.

17. Dulloo AG, Jacquet J \& Girardier L (1997) Poststarvation hyperphagia and body fat overshooting in humans: a role for feedback signals from lean and fat tissues. Am J Clin Nutr 65, 717-723.

18. Mellinkoff SM, Frankland M, Boyle D, et al. (1956) Relationship between serum amino acid concentrations and fluctuations in appetite. J App Physiol 8, 535-538.

19. Millward DJ (1995) A protein-stat mechanism for regulation of growth and maintenance of the lean body mass. Nutr Res Rev 8, 93-120.

20. Fruhbeck G \& Gomez-Ambrosi J (2001) Rationale for the existence of additional adipostatic hormones. FASEB $J \mathbf{1 5}$, 1996-2006.

21. Halford JCG \& Blundell JE (2000) Separate systems for serotonin and leptin in appetite control. Ann Med 32, 222-232.

22. Sáinz N, Rodríguez A, Catalán V, et al. (2009) Leptin administration favors muscle mass accretion by decreasing $\mathrm{FoxO} 3 \mathrm{a}$ and increasing PGC- $1 \alpha$ in ob/ob mice. PLoS One 4, e6808.

23. Wu X, Patki A, Laro-Castro C, et al. (2011) Genes and biochemical pathways in human skeletal muscle affecting resting energy expenditure and fuel partitioning. $J$ Appl Physiol 110, 746-755. 